\title{
Caracterização por FT-IR de Agentes de Cura Utilizados em Resinas Epoxídicas-II-Polimercaptana, Poliaminoamida e Amina Modificada
}

\author{
Benedita M. V. Romão \\ Instituto Tecnológico da Aeronáutica, ITA, CTA \\ Milton F. Diniz, Margarete F. P. Azevedo, Vera L. Lourenço, Luiz C. Pardini, Rita C. L. Dutra \\ Instituto de Aeronáutica e Espaço, CTA \\ Fabrice Burel \\ Institut National des Sciences Appliquées de Rouen, INSA, France
}

\begin{abstract}
Resumo: Amostras de resina epoxídica (EP) curadas com compostos à base de polimercaptana (SH), SH na presença de poliamino amida, e amina modificada, constituindo, respectivamente, os sistemas epoxídicos (SE) 1, 2 e 3, foram preparadas segundo condições estabelecidas pelas curvas de calorimetria exploratória diferencial (DSC) de EP e agentes de cura, ou endurecedores (AC). Estas amostras foram analisadas, antes e após preparação do SE, por espectroscopia no infravermelho com transformada de Fourier (FT-IR) por meio de técnicas de transmissão (pastilha de KBr, pirólise por bico de Bunsen, e pirólise controlada (CONTROLPIR/FT-IR) dentro da faixa de temperatura fornecida pela análise termogravimétrica (TG) dos SE 1, 2 e 3, para a caracterização de cada AC. Para tal fim, as absorções FT-IR do pirolisado líquido obtido pela metodologia CONTROLPIR/FT-IR foram avaliadas, em comparação ao espectro do AC de referência. A caracterização dos agentes de cura foi feita com a técnica TG/FT-IR do gás liberado de cada SE, em comparação ao espectro de referência do produto de degradação do respectivo AC.
\end{abstract}

Palavras-chave: Resina epoxídica, agentes de cura, polimercaptana, amina modificada, pirólise-FT-IR controlada, acoplamento TG/FT-IR.

\section{Characterization by FT-IR of Curing Agents Used in Epoxy Resins}

Abstracts: Samples of epoxy resin (EP) cured with polymercaptans (SH), SH in presence of polyamine amide, and polyamine modified compounds, respectively, SE 1,2 and 3, were prepared according to established conditions by DSC analysis of EP and curing agent (AC) or hardeners. The samples were analyzed by FT-IR transmission techniques (KBr pellets, uncontrolled pyrolysis and controlled pyrolysis-FT-IR) (CONTROLPIR/FT-IR), in the temperature range found from TG analysis of SE 1,2 and 3, for characterizing the AC. The FT-IR absorptions of liquid pyrolysate obtained by CONTROLPIR/ FT-IR were evaluated, in comparison to reference spectrum of AC. The characterization of hardeners was also possible by the analysis of each SE with TG/FT-IR technique, in comparison to the reference spectrum of the degradation product for each AC used.

Keywords: Epoxy resin, curing agent, polymercaptans, polyamine amide, modified amine, controled pyrolysis-FT-IR, coupled TG/FT-IR.

\section{Introdução}

A utilização de diferentes materiais para o desenvolvimento de compósitos com melhores propriedades, demanda e estimula o desenvolvimento de novas metodologias que estabeleçam a relação estrutura/propriedade, fator importante para a aplicação adequada destes sistemas em projetos específicos e de grande porte, como os encontrados na indústria aeroespacial ${ }^{[1]}$.

Os compósitos à base de matrizes orgânicas reforça- das com fibra, são feitos pelo arranjo de mistura de fibra/resina não curada, em moldes apropriados e pela aplicação de calor e pressão $(\mathrm{P})$ ao material por um determinado período até o término da cura. Portanto, valores de temperatura (T), P e a formulação de resina têm um efeito marcante na qualidade do produto final.

É bem conhecido que as propriedades da formulação do sistema epoxídico, isto é, o tipo de agente de cura (endurecedor) e a razão estequiométrica resina/endurecedor, influenciam as propriedades mecânicas do 
compósito $^{[2]}$. Portanto, o conhecimento de agentes de cura é importante para alcançar as propriedades desejadas.

Pesquisas envolvendo a caracterização do comportamento de cura de resinas epoxídicas, por meio de dados térmi$\cos ^{2}$ ou de agentes de cura utilizados em resinas epoxídicas utilizando metodologias FT-IR, vêm sendo desenvolvidas por diferentes pesquisadores da comunidade científica ${ }^{[2,3]}$. Sugita e colaboradores ${ }^{[3]}$ demonstraram que agentes de cura carbonilados são mais facilmente identificáveis, desde que na análise da amostra tal como recebida, na forma de pastilha de $\mathrm{KBr}$, a presença de grupo $\mathrm{C}=\mathrm{O}$ de éster, resultante da reação de cura do anel epóxido/anidrido, seja visualizada. Entretanto, para a separação do agente de cura, utilizaram metodologias complexas, com várias etapas.

Em trabalhos anteriores do nosso grupo ${ }^{[1,4]}$, foi possível demonstrar a separação e caracterização por FT-IR de agentes de cura do tipo anidrido em resina epoxídica e do tipo amido-amina em compósitos, com metodologia simples e rápida, envolvendo técnicas de pirólise controlada - FT-IR (CONTROLPIR/FT-IR), mesmo neste último sistema, onde era esperado um grau de dificuldade maior, por tratar-se de compósito.

Concluiu-se ${ }^{[1,4]}$ que a técnica CONTROLPIR/FT-IR, que analisa o produto líquido resultante da degradação da amostra, aplicada a estes sistemas, em condições adequadas, apresenta em relação às técnicas FT-IR convencionais, a vantagem de separar o agente de cura. Isto permite a sua caracterização, independentemente, de sua função orgânica básica ser indicada ou não na análise da amostra curada. Deste modo, este trabalho se enquadra na linha de pesquisa de caracterização de produtos da indústria aeroespacial, desenvolvida pelo nosso grupo, e serve de apoio a outros projetos específicos que utilizem métodos de caracterização de polímeros.

É também conhecido que o acoplamento de técnicas instrumentais para análise da composição de produtos contribui para a previsão do desempenho dos compósitos, e a crescente manufatura de acessórios específicos para diferentes análises, permite prever um futuro ainda mais promissor na caracterização destes materiais.

O acoplamento da termogravimetria com análise de gás, por FT-IR por exemplo, constitui uma poderosa ferramenta analítica na qual a vantagem de cada técnica é evidenciada ${ }^{[5]}$.

O acoplamento da termogravimetria (TG) e FT-IR é constituído por uma linha de transferência, que consiste em um tubo aquecido que conecta o analisador TG à célula de gás para análise no infravermelho, que também é aquecida ${ }^{[5]}$. Neste caso o fluxo de gás flui para o detetor e não somente uma fração do gás ${ }^{[5]}$.

As vantagens deste tipo de acoplamento estão na rapidez de preparação de amostra e flexibilidade de aplicação.

Deste modo, neste trabalho, os resultados da técnica CONTROLPIR/FT-IR para os produtos líquidos de pirólise dos sistemas epoxídicos contendo resina diglicidiléter de bisfenol A (DGEBA) e 3 diferentes agentes de cura, polimercaptana, poliaminoamida e amina modificada, foram aliados aos dados dos produtos gasosos fornecidos pela técnica TG/FT-IR, dados estes, obtidos por pesquisadores franceses do INSA (Institut des Sciences Appliquées), que estão trabalhando em conjunto com nosso grupo.

Como é conhecido, resinas epoxídicas constituem as matrizes poliméricas mais utilizadas em compósitos encontrados nas indústrias, automobilística, de construção e aeroespacial ${ }^{[6,7]}$, sendo que o pré-polímero mais usado é DGEBA.

A cura da resina com diferentes agentes, tanto à temperatura ambiente como a altas temperaturas, depende dos produtos de partida utilizados no processo e das propriedades esperadas para o produto final ${ }^{[2]}$. Anidridos e aminas são os agentes mais utilizados. Entretanto, polimercaptanas são também utilizadas em compósitos com resinas epoxídicas ou fenólicas, e outros agentes de última geração à base de aminas modificadas ou poliaminoamida são tradicionais no mercado, e apresentam alta reatividade e resistência química ${ }^{[8]}$.

Diante do exposto, julgou-se atrativo o desenvolvimento de uma metodologia que identificasse o agente de cura, em sistemas epoxídicos (DGEBA + AC) contendo polimer-captana, amina modificada ou polimercaptanas/poliaminoamida como $\mathrm{AC}$, que servissem de base para este tipo de determinação em compósitos contendo composição similar.

\section{Experimental}

\section{Materiais}

Amostras de resina EP à base de diglicidiléter de bisfenolA (DGEBA). GENOPOXY 190 - 859361 foram curadas com polimercaptana (Capcure 3-800), ou Capcure 3-800 e poliaminoamida padrão (Versamid 125), ou aduto de amina modificada (Versamine CEX 13.320). Estes materiais foram gentilmente cedidos pela COGNIS S. A.

\section{Estruturas químicas dos agentes de cura}

\section{aCapcure $^{(\mathrm{R})}$ - marca registrada de agente de cura para} resina epoxídica

Uma típica polimercaptana apresenta uma estrutura química similar a:

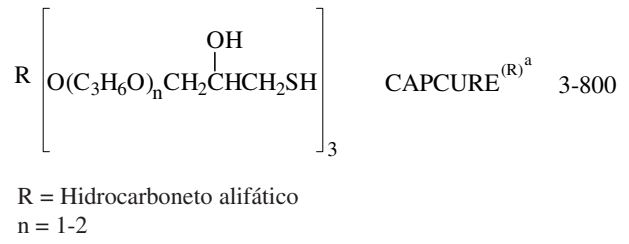

\section{Versamine CEX 13320}

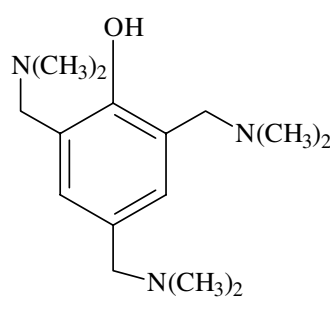

Aduto de Amina 


\section{Versamid 125 (poliaminoamida)}

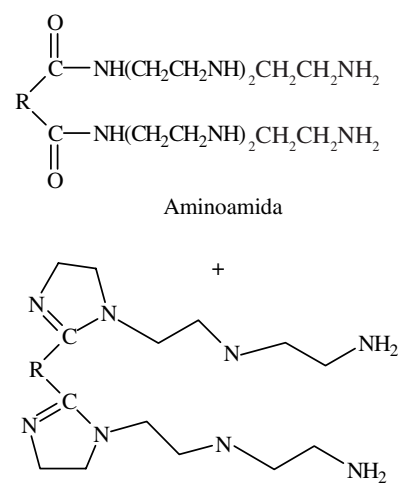

Imidazolina

\section{Análise DSC das misturas EP/AC}

Para a obtenção das curvas DSC foi utilizado o analisador térmico TA2100 TA INSTRUMENTS com módulo DSC-910. As amostras EP/AC, nas proporções EP/Capcure 3-800 (1:1), EP/Capcure 3-800/Versamid 125 (100:10:1) e EP/Versamine CEX 13320 (100:40) foram preparadas, misturadas, homogeneizadas e analisadas imediatamente por DSC.

As amostras foram colocadas em porta-amostras herméticas de alumínio e aquecidas a $10^{\circ} \mathrm{C} / \mathrm{min}$, da T ambiente até completar a exoterma de cura $\left(180,120\right.$ e $200^{\circ} \mathrm{C}$ respectivamente), sob atmosfera de nitrogênio $\left(<5 \mathrm{ppm} \mathrm{H}_{2} \mathrm{O}\right.$, $50 \mathrm{~mL} / \mathrm{min}$ ). As amostras EP/ Capcure 3-800/ Versamid 125 (100:10:1) e EP/Versamine CEX 13320 (100:40) foram resfriadas bruscamente com nitrogênio líquido e aquecidas novamente até 100 e $150{ }^{\circ} \mathrm{C}$, respectivamente, até a região de transição vítrea ser observada. Foi analisada 1 alíquota de cada amostra.

\section{Preparação dos SE}

Após a análise DSC, os sistemas epoxídicos foram curados, em estufa, nas seguintes condições: EP/Capcure 3-800 (1:1) (SE 1) - cura processada a $122{ }^{\circ} \mathrm{C}, 1 \mathrm{~h}$; EP/Capcure 3-800/Versamid 125 (100:10:1) (SE2), 3 dias a $125^{\circ} \mathrm{C}$; EP/Versamine CEX 13320 (100:40) (SE 3) - $1 \mathrm{~h}$ a $50{ }^{\circ} \mathrm{C}+1 \mathrm{~h}$ a $60{ }^{\circ} \mathrm{C}+1 \mathrm{~h}$ a $90{ }^{\circ} \mathrm{C}$.

\section{Análise TG de EP, AC e SE}

Para a obtenção das curvas TG foi utilizado o analisador térmico TA2100 TA Instrument com módulo TGA-951. As amostras EP, AC e SE ( 20 mg) foram colocadas em suporte de platina, aquecidas a $10^{\circ} \mathrm{C} / \mathrm{min}$ até $700^{\circ} \mathrm{C}$, em atmosfera de ar sintético $(50 \mathrm{~mL} / \mathrm{min})$. Foram analisadas 2 alíquotas para cada amostra.

\section{Análise por FT-IR de EP, AC e SE}

Para a obtenção dos espectros foi utilizado o FT-IR SPECTRUM 2000 PERKINELMER com as seguintes condições: resolução $4 \mathrm{~cm}^{-1}$, ganho 1 , região espectral 4000 a $400 \mathrm{~cm}^{-1} \mathrm{e} 40$ varreduras. Amostras de resina EP GENOPOXY 190 curadas com Capcure 3-800, Versamid 125 e Versamine

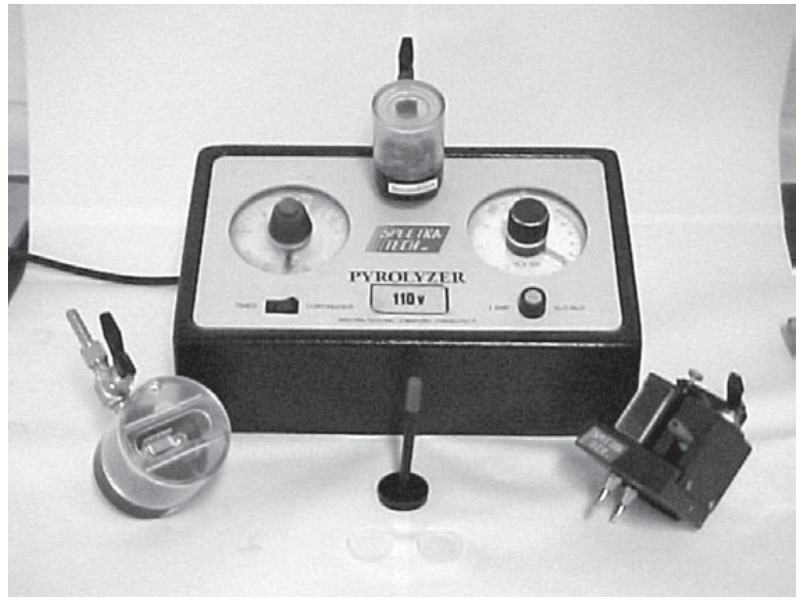

Figura 1. Aparelhagem de pirólise controlada.

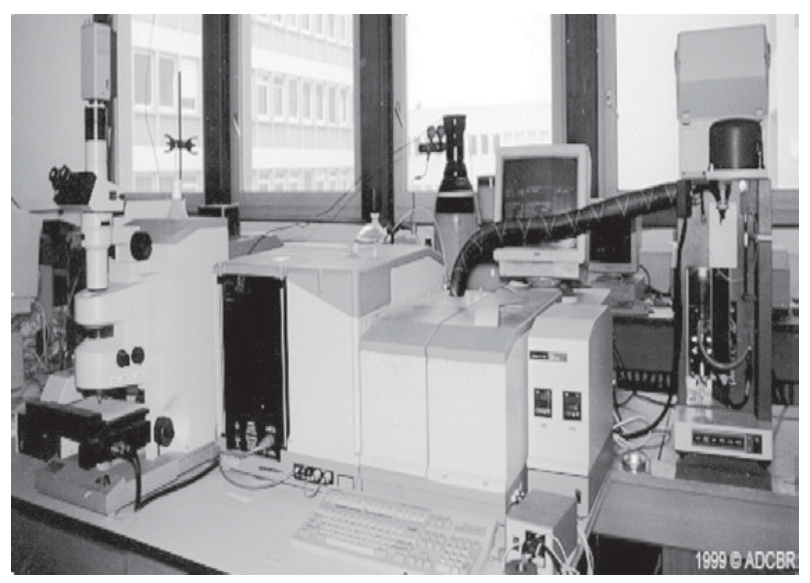

Figura 2. Aparelhagem TG/FT-IR.

CEX 13320 (COGNIS S.A) foram analisadas na forma de pastilha de $\mathrm{KBr}$ (1:400 mg) e também pirolisadas em bico de Bunsen, sendo analisadas como filmes líquidos. Para a metodologia CONTROLPIR/FT-IR, cerca de 0,5 g de amostra dos SE foram também pirolisadas em aparelho SPECTRATECH modelo PY-2 (Figura 1), utilizando-se suporte adequado à análise da fase condensada do material. Os produtos de pirólise foram obtidos em diferentes condições de tempo e temperatura (SE $1-400{ }^{\circ} \mathrm{C} / 10 \mathrm{~min}$; SE 2 e SE 3 $350^{\circ} \mathrm{C} / 10 \mathrm{~min}$ ), sendo analisados como filmes líquidos (SE 1 e SE 3). O pirolisado da SE 2 foi recolhido em pastilha de $\mathrm{KBr}$ (200 mg de KBr).

\section{Análise por TG/FT-IR da resina EP, AC e SE}

Para a obtenção das curvas térmicas e espectros foi utilizado o acoplamento TG7/FT-IR SPECTRUM 2000 PERKINELMER (Figura 2) com as seguintes condições: as amostras foram colocadas em suporte de platina, aquecidas entre 10 e $40^{\circ} \mathrm{C} / \mathrm{min}$ de 150 até $700^{\circ} \mathrm{C}$, em atmosfera de ar sintético $(50 \mathrm{~mL} / \mathrm{min})$. Foram analisadas 2 alíquotas para cada amostra. A identificação dos produtos gasosos foi feita pelo FT-IR acoplado. 


\section{Resultados e Discussão}

\section{Características e propriedades dos materiais utilizados/ Desenvolvimento da metodologia envolvendo técnicas IR/ $D S C / T G / T G-I R$}

Amostras de EP curadas com compostos à base de polimercaptana $(\mathrm{SH}), \mathrm{SH}$ na presença de poliamino amida, e amina modificada, constituindo, respectivamente, os sistemas epoxídicos SE 1, 2 e 3, foram preparadas, segundo condições definidas a partir das curvas DSC das misturas EP/AC, que estabeleceram o perfil de cura para os sistemas epoxídicos.

A metodologia escolhida para a identificação dos agentes de cura nos SE foi a espectroscopia FT-IR, que já apresentou bons resultados para esse tipo de determinação, porém com a utilização de metodologia mais complexa ${ }^{[3]}$. A técnica IR utilizada nesta metodologia, desenvolvida em nossos laboratórios ${ }^{[1,4]}$, foi a pirólise (degradação térmica) controlada (dados de tempo, $\mathrm{t}$, associados à faixa de $\mathrm{T}$ no pirolisador e à quantidade de vapores adequados à análise IR) e temperatura ( $\mathrm{T}$ de degradação fornecida pela análise TG), desde que para a adequada separação de componentes, é necessário o controle de tais parâmetros. Por esta técnica foram analisados os pirolisados líquidos dos materiais.

\section{Análise por FT-IR de EP, AC}

Inicialmente, a resina $\mathrm{EP}$ e os agentes $\mathrm{AC}$ foram analisados por meio de técnicas IR de transmissão para que as absorções dos materiais de partida fossem conhecidas.

Desde que bandas IR de resinas epoxídicas são amplamente discutidas na literatura ${ }^{[9]}$, nesse trabalho incluiu-se as absorções IR dos agentes à base de polimercaptana, poliaminoamida e amina modificada, atribuídas aos seus respectivos modos vibracionais ${ }^{[10]}$. (Tabelas 1 a 3 ).

Como características de cada agente, pode ser destacado que polimercaptanas são, normalmente, utilizadas onde se necessita cura rápida; poliaminoamidas são provenientes de uma reação de poliamida com excesso estequiométrico de amina. Com isso obtém-se um produto que terá características intermediárias entre uma poliamida e uma amina alifática, adequado em casos onde sejam requeridas maior tenacidade e adesividade com melhor fluidez e maior reatividade. Adutos de aminas são resultantes da mistura de resina epoxídica ou diluente reativo com um excesso de amina. Com isso obtémse uma cadeia maior e menos voláteis, e consequentemente, possuem uma relação de mistura menos crítica, curam mais rapidamente e possuem menor toxicidade.

\section{Análise DSC das misturas EP/AC}

As curvas DSC das misturas SE 1, 2 e 3 estão na Figura 3. A curva DSC da mistura SE 1 (EP/Capcure 3-800 1/1) mostra um pico exotérmico bem definido entre 75 e $150{ }^{\circ} \mathrm{C}$, com máximo em $122{ }^{\circ} \mathrm{C}$, que deve ser atribuído à cura do sistema epoxídico.

As curvas DSC das misturas SE 2 e SE 3 apresentam exotermas de cura com temperatura máxima em 92 e $89^{\circ} \mathrm{C}$, respectivamente, sendo que a Tabela 4 contém os valores de $\mathrm{T}$ inicial $\left(\mathrm{T}_{\mathrm{i}}\right)$, $\mathrm{T}$ de início extrapolado $\left(\mathrm{T}_{\text {onset }}\right)$ e do máximo do pico $\left(\mathrm{T}_{\mathrm{p}}\right)$ destes SE.

As misturas SE 2 e 3 foram resfriadas e aquecidas novamente. A ausência de pico exotérmico e a presença de transição vítrea $\left(\mathrm{T}_{\mathrm{g}}\right)$ mostra que as misturas estão totalmente curadas.

Tabela 1. Bandas características de Capcure 3-800, atribuídas aos modos vibracionais típicos ${ }^{[10]}$.

\begin{tabular}{lcc}
\hline $\begin{array}{l}\text { Número de } \\
\text { onda }\left(\mathbf{c m}^{-1}\right)\end{array}$ & $\begin{array}{c}\text { Grupo } \\
\text { funcional }\end{array}$ & $\begin{array}{c}\text { Modo } \\
\text { vibracional }\end{array}$ \\
\hline 2559 & $\mathrm{SH}$ & $\mathrm{n}$ \\
1455 & $\mathrm{CH}_{2} \mathrm{e} \mathrm{CH} \mathrm{CH}_{3}$ & $\delta_{\mathrm{s}} \mathrm{CH}_{2}$ \\
& & $\delta_{\mathrm{a}} \mathrm{CH}_{3}$ \\
1416 & $\mathrm{CH}_{3} \mathrm{~S}-$ & $\delta_{\mathrm{a}}$ \\
1375 & $\mathrm{CH}_{3}$ & $\delta_{\mathrm{s}}$ \\
1299 & $\mathrm{CH}_{3} \mathrm{~S}-$ & $\delta_{\mathrm{s}}$ \\
1106 & $\mathrm{C}-\mathrm{O}, \mathrm{S}-\mathrm{S}$ & $\mathrm{V}$ \\
\hline (deformação axial ou estiramento); $\delta_{\mathrm{s}}$ (deformação angular simétrica); \\
(deformąão angular assimétrica).
\end{tabular}

Tabela 2. Bandas características de Versamid 125, atribuídas aos modos vibracionais típicos ${ }^{[10]}$.

\begin{tabular}{|c|c|c|}
\hline $\begin{array}{l}\text { Número de } \\
\text { onda }\left(\mathrm{cm}^{-1}\right)\end{array}$ & $\begin{array}{c}\text { Grupo } \\
\text { funcional }\end{array}$ & $\begin{array}{c}\text { Modo } \\
\text { vibracional }\end{array}$ \\
\hline 3289 & NH & $\mathrm{n}$ \\
\hline 2919 & $\mathrm{CH}_{2}$ & $v_{a}$ \\
\hline 2853 & $\mathrm{CH}_{2}$ & $v_{\mathrm{s}}$ \\
\hline 1651 & $\mathrm{C}=\mathrm{O}$ amida $\mathrm{I}$ & $v$ \\
\hline 1645 & $\mathrm{NH}_{2}$ & d \\
\hline 1557 & $\begin{array}{c}\mathrm{C}-\mathrm{N} \text { e } \mathrm{NH} \text { (amida II), } \\
\mathrm{C}=\mathrm{N}\end{array}$ & $\begin{array}{c}\delta \mathrm{NH}, v \mathrm{CN} \\
\quad v \mathrm{C}=\mathrm{N}\end{array}$ \\
\hline 1456 & $\mathrm{CH}_{2}$ e $\mathrm{CH}_{3}$ & $\begin{array}{l}\delta_{\mathrm{s}} \mathrm{CH}_{2} \\
\delta_{\mathrm{a}} \mathrm{CH}_{3}\end{array}$ \\
\hline 1366 & $\mathrm{CH}_{3}$ & ${ }^{\mathrm{a}} \delta_{\mathrm{s}}{ }^{3}$ \\
\hline 722 & $\mathrm{NH}_{2}, \mathrm{NH}, \mathrm{CH}_{2}$ & $\omega \mathrm{NH}_{2}, \mathrm{NH}, \rho \mathrm{CH}_{2}$ \\
\hline
\end{tabular}

Tabela 3. Bandas características de Versamine CEX 13320, atribuídas aos modos vibracionais típicos ${ }^{[10]}$.

\begin{tabular}{lcc}
\hline $\begin{array}{l}\text { Número de } \\
\text { onda }\left(\mathbf{c m}^{-1}\right)\end{array}$ & $\begin{array}{c}\text { Grupo } \\
\text { funcional }\end{array}$ & $\begin{array}{c}\text { Modo } \\
\text { vibracional }\end{array}$ \\
\hline 3355 & $\mathrm{NH}$ & $\mathrm{v}_{\mathrm{a}}$ \\
3292 & $\mathrm{NH}$ & $\mathrm{v}_{\mathrm{s}}$ \\
3026 & $\mathrm{C}-\mathrm{H}$ & $\mathrm{v}$ \\
2960 & $\mathrm{CH}_{3}$ & $\mathrm{v}_{\mathrm{a}}$ \\
1610 & $\mathrm{C}-\mathrm{C}$ aromático & $\mathrm{v}$, \\
1591 & $\mathrm{NH}_{2}$ & $\delta$ \\
1515 & $\mathrm{C}-\mathrm{C}$ aromático $_{15}$ & $\mathrm{v}$ \\
1463 & $\mathrm{CH}_{2}$ e CH & $\delta_{\mathrm{a}} \mathrm{e} \delta_{\mathrm{s}}$ \\
1362 & $\mathrm{CH}_{3}$ & $\delta_{\mathrm{s}}$ \\
1299 & $\mathrm{C}-\mathrm{N}_{12}$ & $\mathrm{v}$ \\
831 & $\mathrm{C}-\mathrm{OC}$ & $\mathrm{v}$ \\
790 & Epóxido terminal, & Vibração do anel \\
\hline
\end{tabular}


Os valores de $\mathrm{T}_{\mathrm{g}}$ encontrados estão na Tabela 4. A amostra SE 2 foi resfriada e aquecida duas vezes para confirmação da $\mathrm{T}_{\mathrm{g}}$ observada, pois ocorre em temperatura mais baixa que o esperado para sistemas epoxídicos.

Pode ser observado da Tabela 4, que as Tg das misturas curadas não mostraram uma tendência definida em relação ao pico de cura, ocorrendo ora na região da $\mathrm{T}_{\mathrm{p}}$, ora muito abaixo da região de cura.

Os dados das curvas DSC foram utilizados para processar a cura dos sistemas, conforme já citado na parte experimental. No caso do SE 2, a amostra ainda estava flexível após a análise DSC e, devido à semelhança do sistema com a SE 1, a cura foi realizada em $125^{\circ} \mathrm{C}$, para garantir a reação

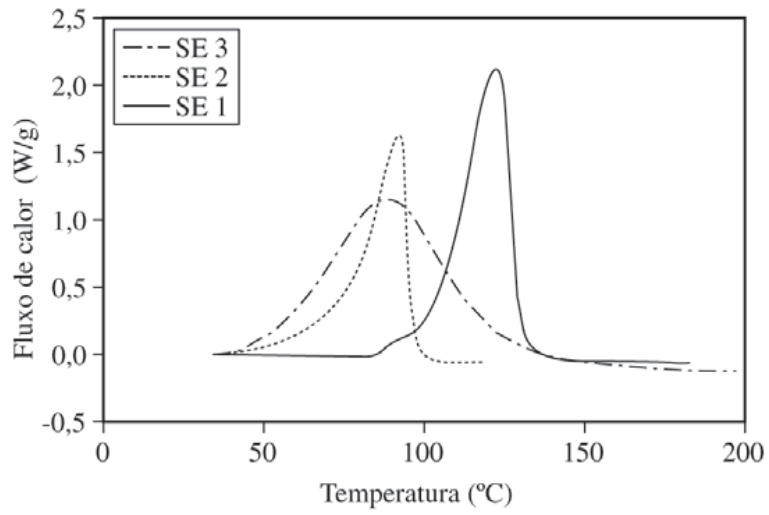

Figura 3. Curva DSC das misturas SE 1,2 e 3.

Tabela 4. Resultados da análise DSC das misturas EP/AC

\begin{tabular}{|c|c|c|c|c|}
\hline $\begin{array}{l}\mathrm{EP} / \mathrm{AC} \\
\left({ }^{\circ} \mathrm{C}\right)\end{array}$ & $\begin{array}{l}\mathrm{T}_{\text {inicial }} \\
\left({ }^{\circ} \mathrm{C}\right)\end{array}$ & $\begin{array}{l}\mathrm{T}_{\text {onset }} \\
\left({ }^{\circ} \mathrm{C}\right)\end{array}$ & $\begin{array}{l}\mathrm{T}_{\text {máxima }} \\
\left({ }^{\circ} \mathrm{C}\right)\end{array}$ & $\mathrm{T}_{\mathrm{g}}$ \\
\hline EP/Capcure 3-800/ & & & & 12 \\
\hline Versamid 125 (SE 2) & 35 & 74 & 92 & 13 \\
\hline $\begin{array}{l}\text { EP/Versamine CEX } 13320 \\
\text { (SE 3) }\end{array}$ & 37 & 53 & 89 & 80 \\
\hline
\end{tabular}

total. A $\mathrm{T}_{\mathrm{g}}$ da placa obtida, ainda assim, foi igual a $16{ }^{\circ} \mathrm{C}$, ou seja, continuou flexível, o que talvez possa ser explicado pela diferente proporção EP/AC.

\section{Análise TG de EP, AC e SE}

A Tabela 5 mostra os intervalos de temperatura $(\Delta \mathrm{T})$ onde foi observada perda de massa $(\Delta \mathrm{m})$, valores entre parênteses, nas curvas TG de EP, AC e SE, valores médios de duas alíquotas.

Todas as amostras deixaram resíduo inferior a $1 \%$ ao final do aquecimento. O Capcure 3-800, em $320^{\circ} \mathrm{C}$, apresentou um aumento de temperatura na curva de tempo/temperatura, indicando forte e rápida reação exotérmica da amostra.

De uma forma geral, todas as amostras perdem massa em três etapas. As curvas TG dos SE não são a superposição dos puros, o que não permite identificar em qual região estão sendo liberados os AC pois EP e AC perdem massa em regiões próximas e, após a cura, o mecanismo de degradação deve ser alterado.

A curva termogravimétrica derivada (DTG) é proporcional à velocidade de reação, sendo que a temperatura do máximo do pico corresponde ao ponto onde a reação é mais rápida. Esta temperatura é mostrada na Tabela 5 para a etapa onde a perda de massa é mais intensa, informação utilizada para estabelecer a temperatura a ser utilizada nas técnicas CONTROL-PIR ou TG/FT-IR, com a liberação de maior quantidade de gases em menor tempo.

\section{Análise por FT-IR (CONTROL-PIR) doS SE}

Conforme citado, dados de análise TG observados para a resina Genepoxy 190 e os agentes de cura Capcure 3-800, Versamid 125 e Versamine CEX13320, que mostraram T de pico DTG entre 350 e $400{ }^{\circ} \mathrm{C}$, serviram de base para o estabelecimento da T para a análise dos SE. O tempo de $10 \mathrm{~min}$ foi adotado para todas as placas, desde que é o adequado para esta faixa de temperatura, visando a obtenção de quantidade de amostra suficiente para análise.

Tabela 5. Resultados da análise TG de EP, AC e SE.

\begin{tabular}{|c|c|c|c|c|c|c|}
\hline Amostra & $\Delta \mathrm{T}\left({ }^{\circ} \mathrm{C}\right)$ & $(\Delta \mathrm{m})(\%)$ & $\Delta \mathrm{T}\left({ }^{\circ} \mathrm{C}\right)$ & $(\Delta \mathrm{m})(\%)$ & $\Delta \mathrm{T}\left({ }^{\circ} \mathrm{C}\right)$ & $(\Delta \mathrm{m})(\%)$ \\
\hline Resina & - & - & $\begin{array}{c}200-346 \\
340^{\mathrm{a}}\end{array}$ & $(85)$ & $\begin{array}{l}346-420 \\
420-600^{c}\end{array}$ & $\begin{array}{l}(8) \\
(4)\end{array}$ \\
\hline $\begin{array}{l}\text { Capcure } \\
3-800\end{array}$ & $26-206$ & (2) & $\begin{array}{c}206-370 \\
320^{\mathrm{a}, \mathrm{b}}\end{array}$ & (93) & $370-600^{c}$ & (4) \\
\hline$\overline{\mathrm{SE} 1}$ & $28-200$ & (2) & $\begin{array}{c}200-432 \\
350^{\mathrm{a}}\end{array}$ & (77) & $432-600$ & (20) \\
\hline Versamid 125 & $28-230$ & (9) & $\begin{array}{c}230-468 \\
450^{\mathrm{a}}\end{array}$ & (75) & $468-600^{c}$ & (15) \\
\hline$\overline{\text { SE2 }}$ & - & - & $\begin{array}{c}200-490 \\
410^{\mathrm{a}}\end{array}$ & (75) & $490-620^{c}$ & (24) \\
\hline $\begin{array}{l}\text { Versamine } \\
\text { CEX 13-320 }\end{array}$ & - & - & $\begin{array}{c}26-300 \\
200^{\mathrm{a}}\end{array}$ & (81) & $300-600^{c}$ & (18) \\
\hline SE3 & - & - & $\begin{array}{c}100-450 \\
350^{\mathrm{a}}\end{array}$ & (67) & $450-650^{c}$ & (33) \\
\hline
\end{tabular}




\section{SE 1}

A Figura 4, que inclui espectros do sistema EP/Capcure3-800 (SE 1), amostra em forma de pastilha de $\mathrm{KBr}$ (a); pirólise em bico de Bunsen (b) e controlada a $400{ }^{\circ} \mathrm{C} / 10 \mathrm{~min}$ (c) mostra que a técnica CONTROLPIR/FT-IR é adequada para a identificação do Capcure-3-800, desde que as bandas desta polimercaptana (d), em 1416 e $1299 \mathrm{~cm}^{-1}\left(\delta \mathrm{CH}_{3} \mathrm{~S}-\right)$ e $1106 \mathrm{~cm}^{-1}$ ( $v \mathrm{C}-\mathrm{O}$ e/ou S-S) foram confirmadas. É conhecido $^{[11]}$ que a banda de SH em $2600 \mathrm{~cm}^{-1}$ é de intensidade variável e de difícil observação. Portanto, as bandas citadas, que se encontram na região de impressão digital, já são suficientes para a caracterização do agente de cura.

A amostra em forma de pastilha mostra somente que a resina está curada devido à ausência da banda em $917 \mathrm{~cm}^{-1} \mathrm{de}$ grupo epóxido terminal, que constitui a principal diferença entre os espectros de resinas epoxídicas curadas ou não ${ }^{[9]}$. Como não é observada banda de $\mathrm{C}=\mathrm{O}$ no espectro obtido pela técnica pastilha, pode-se somente citar que a amostra não foi curada com anidrido ou composto carbonilado ${ }^{[3,4]}$. A pirólise em bico de Bunsen mostra ainda absorções da resina epoxídica entre 1300 a $930 \mathrm{~cm}^{-1}$ (vCOC). Portanto, a técnica CONTROLPIR/FT-IR apresenta vantagem em relação às convencionais, pois sem requintes de preparação de amostra, é capaz de separar o agente de cura, de modo a facilitar a sua caracterização. Isto pode ser visualizado por meio das bandas assinaladas no espectro FT-IR (c) do pirolisado da SE 1, em comparação ao (d), relativo ao agente de cura Capcure 3-800.

\section{SE 2}

A mesma metodologia foi usada para análise do sistema epoxídico SE 2 curado com Capcure 3-800 em presença de pequena quantidade (1\% em relação ao Capcure 3-800) de composto à base de poliaminoamida (Versamid 125). As condições e espectros do SE 2 analisado segundo técnicas diferentes, em comparação ao espectro do agente de cura

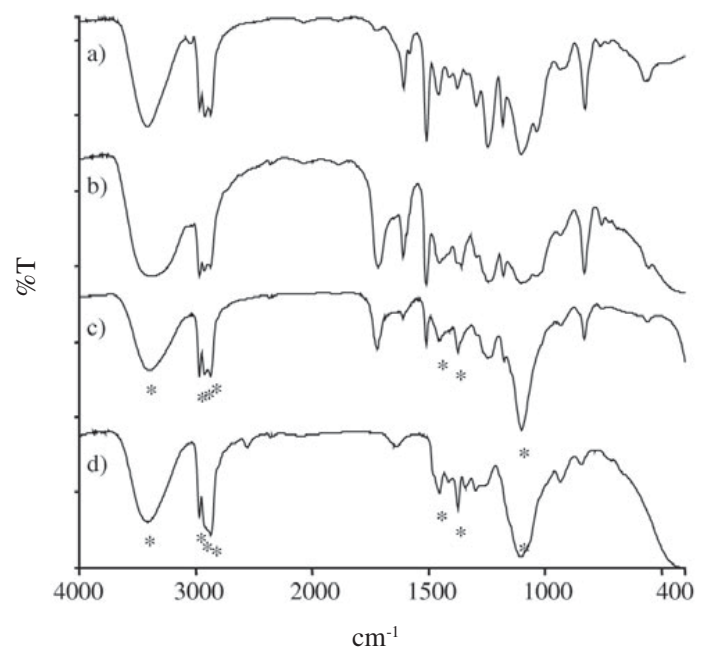

Figura 4. Espectros FT-IR do SE 1, segundo as técnicas: a) Pastilha de $\mathrm{KBr}$ 1:400 mg; b) Pirólise em bico de Bunsen; c) Pirólise controlada a $400{ }^{\circ} \mathrm{C} / 10 \mathrm{~min} ; \mathrm{d}$ ) agente de cura Capcure 3-800 (filme líquido). são mostrados na Figura 5. No espectro (técnica em pastilha) do sistema curado não é observada a absorção de $\mathrm{C}=\mathrm{O}$, como esperado, desde que o agente não é anidrido ${ }^{[3]}$. Entretanto, pode ser observado que as absorções observadas na região de impressão digital do espectro do pirolisado obtido a $350{ }^{\circ} \mathrm{C} / 10$ min caracterizam a presença do agente de cura Capcure, mostrando que a pirólise controlada nestas condições é a mais adequada à análise deste agente na presença de Versamid na quantidade usada.

A visualização das absorções do Capcure 3-800 é esperada desde que está em maior proporção.

\section{SE 3}

A metodologia foi, também, usada para o SE 3. As condições e espectros do SE 3 analisado segundo técnicas diferentes, em comparação ao espectro do agente de cura são mostrados na Figura 6. Pode ser observado que as condições utilizadas para a pirólise controlada revelam melhor as absorções do agente amínico (regiões de NH e C-N). A similaridade de algumas regiões com absorções de resina epoxídica é esperada desde que este agente é um aduto.

Portanto, as Figuras 4 a 6 mostram que a metodologia CONTROL-PIR/FT-IR desenvolvida em nossos laboratórios é viável para estudo de agentes de cura de resinas epoxídicas à base de polimercaptanas, puro ou em presença de pequena quantidade de poliaminoamidas, e adutos de aminas, em condições específicas.

Dando continuidade à pesquisa, análises de sistemas epoxídicos com diferentes agentes de cura pela técnica TG/FT-IR foram realizadas no INSA, em cooperação com pesquisadores franceses. Os resultados são discutidos a seguir.

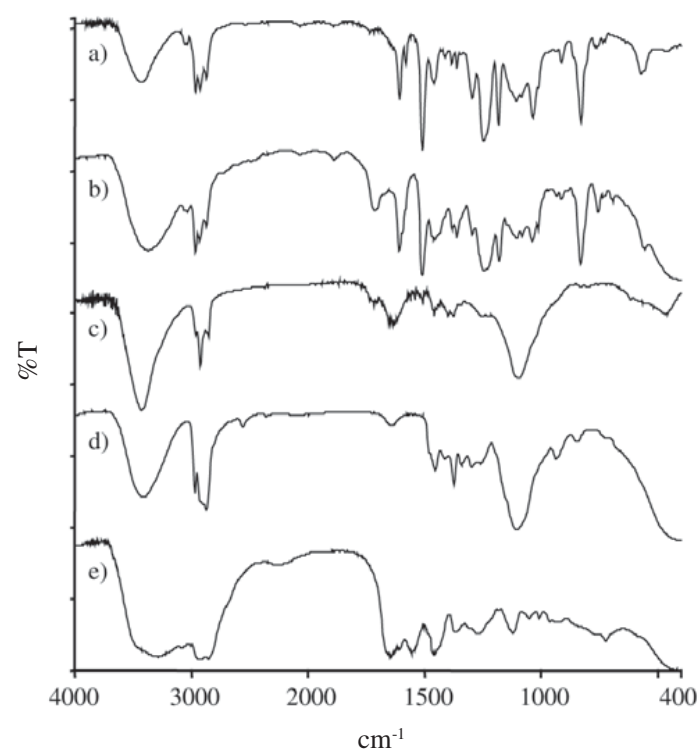

Figura 5. Espectros FT-IR do SE 2 segundo as técnicas: a) Pastilha de $\mathrm{KBr}$ 1:400 mg; b) Pirólise em bico de Bunsen; c) Pirólise controlada a 350 ${ }^{\circ} \mathrm{C} / 1$ min d) Capcure 3-800; (filme líquido) e) agente de cura Versamid 125 (filme líquido). 


\section{Análise por TG/FT-IR da resina EP e AC}

A Figura 7 revela as absorções FT-IR dos produtos de degradação da resina epoxídica Genepoxy 190, Capcure 3-800, Versamid 125 e Versamine CEX 13320. Pode ser observado nesta Figura 7 que os espectros apresentam diferenças em determinadas regiões características, sugerindo que as bandas do produto de degradação da resina não influenciará, de modo significativo na investigação dos agentes de cura nos sistemas epoxídicos.

As principais absorções do produto de degradação da resina epoxídica Genepoxy 190 estão em 3015, 1731 e

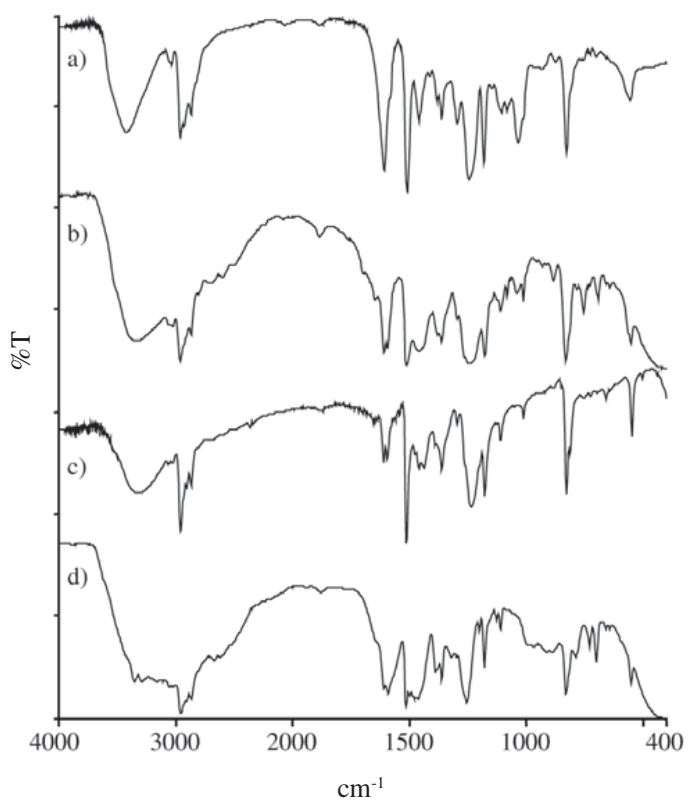

Figura 6. Espectros FT-IR do SE 3, segundo as técnicas: a) Pastilha de $\mathrm{KBr}$ 1:400 mg; b) Pirólise em bico de Bunsen; c) Pirólise controlada a $350{ }^{\circ} \mathrm{C} / 10 \mathrm{~min}$; d) agente de cura Versamine CEX 13320 (filme líquido).

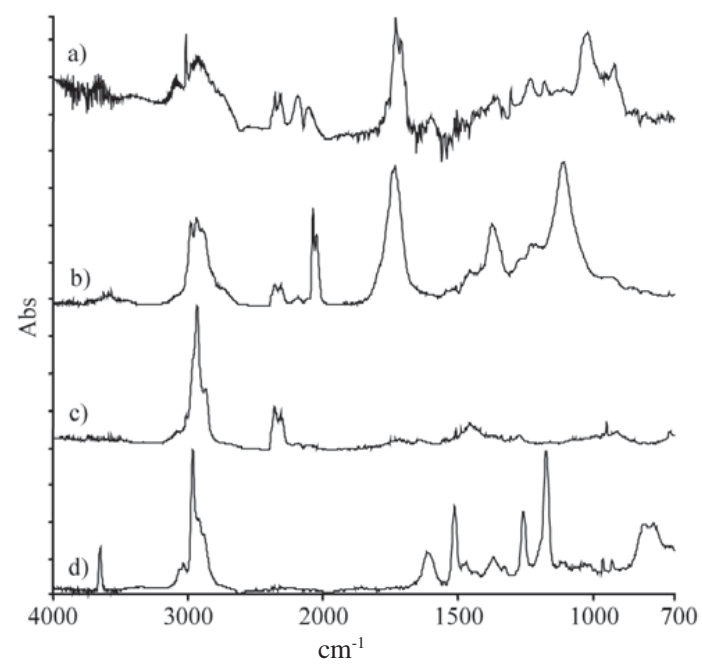

Figura 7. Espectros FT-IR dos produtos de degradação da resina EP e AC: a) EP, b) Capcure 3-800, c) Versamid 125, d) Versamine CEX 13320.
$1018 \mathrm{~cm}^{-1}$, e podem ser, provavelmente, atribuídas ${ }^{[9,12]}$, aos grupos $\mathrm{C}-\mathrm{H}$ aromático, $\mathrm{C}=\mathrm{O}$ e C-O (éter aromático), respectivamente, de acordo com processos de degradação estabelecidos para resinas epoxídicas e sistemas epoxídicos por Dyakonov ${ }^{[12]}$.

As principais absorções do produto de degradação do Capcure 3-800, associadas a uma provável atribuição ${ }^{[10]}$, estão em: $3566 \mathrm{~cm}^{-1}\left(\mathrm{H}_{2} \mathrm{O}\right), 2978$ e $2937 \mathrm{~cm}^{-1}$ (grupo $\mathrm{CH}$ ), 2355 $\mathrm{cm}^{-1}$ (grupo $\mathrm{CO}_{2}$ ), $1732 \mathrm{~cm}^{-1}$ (grupo $\mathrm{C}=\mathrm{O}$ ) e $1110 \mathrm{~cm}^{-1}$ (grupo C-S).

As principais absorções do produto de degradação do Versamid 125 estão em 2932, 2357, 1456, e $949 \mathrm{~cm}^{-1}$, que podem ser, provavelmente, atribuídas à presença de $\mathrm{CH}, \mathrm{CO}_{2}, \mathrm{H}_{2} \mathrm{O} \mathrm{e}$ $\mathrm{NH}_{3}$, respectivamente, de acordo com Regeers et al..$^{[13]} \mathrm{e}$ Hornsby et al. ${ }^{[14]}$ que estudaram espectros de gases e produtos de degradação de poliamidas, em temperaturas específicas.

As principais absorções do produto de degradação do Versamine 13320 estão em 3653, 2965, 1613, 1513, 1258 e $1175 \mathrm{~cm}^{-1}$, que podem ser, provavelmente, atribuídas à presença de $\mathrm{H}_{2} \mathrm{O}\left(3653\right.$ e $\left.1613 \mathrm{~cm}^{-1}\right), \mathrm{CH}\left(2965 \mathrm{~cm}^{-1}\right)$, $\mathrm{NH}\left(1513 \mathrm{~cm}^{-1}\right)$ e CN $\left(1258\right.$ e $\left.1175 \mathrm{~cm}^{-1}\right)$.

\section{Análise por TG/FT-IR dos SE \\ SE 1}

A Figura 8, que inclui os espectros de Capcure 3-800 e da SE 1, revela que é possível identificar o agente de cura neste sistema epoxídico, uma vez que as absorções do produto de degradação do agente são observadas no espectro do SE 1.

\section{SE 2}

A Figura 9, de forma similar ao SE1, revela que é possível identificar no sistema epoxídico SE2, os agentes de cura Capcure 3-800 e Versamid 125, embora este último apresente um número pequeno de absorções, e com intensidade fraca. As absorções do agente Versamid 125 são melhor visualizadas

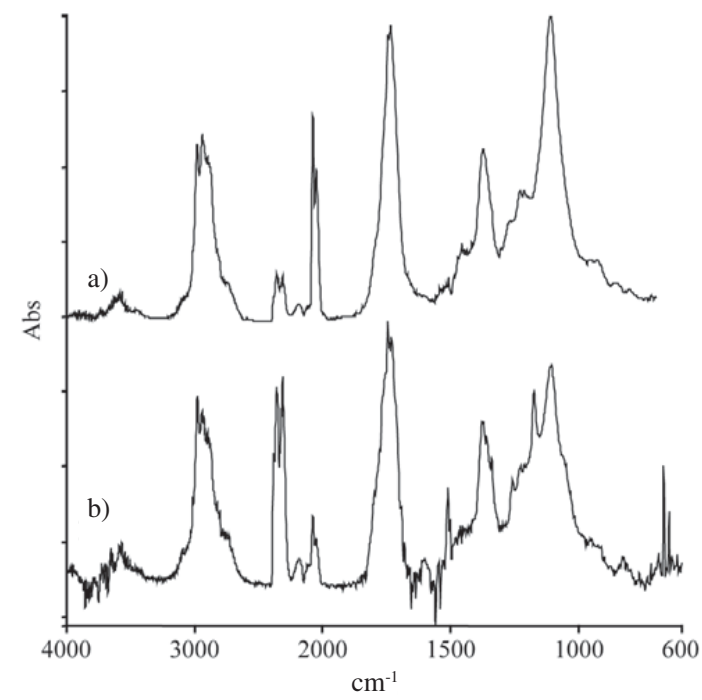

Figura 8. Espectros FT-IR dos produtos de degradação de Capcure 3-800 e SE 1. a) Capcure 3-800, b) SE 1. 


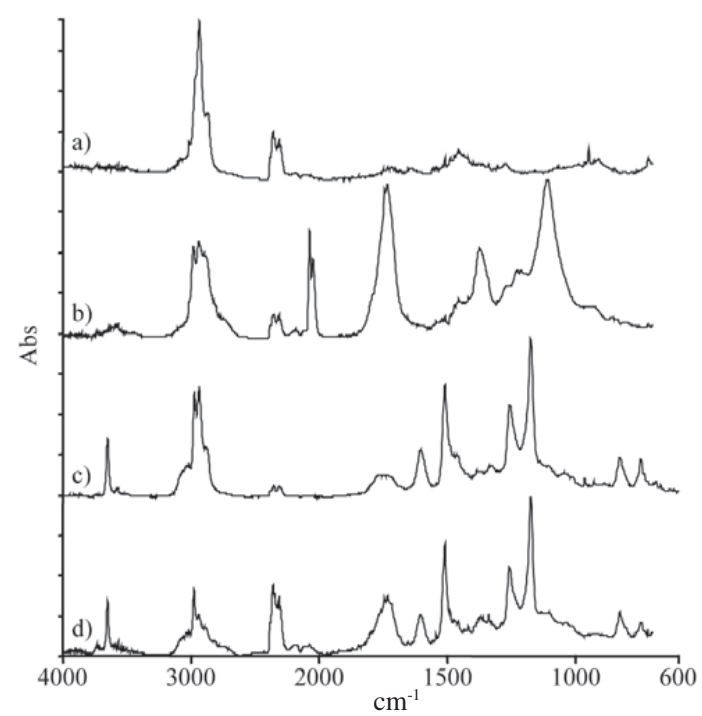

Figura 9. Espectros FT-IR dos produtos de degradação de a) Versamid 125, b) Capcure 3-800, c) sistema epoxídico contendo Versamid 125 e d) SE 2.

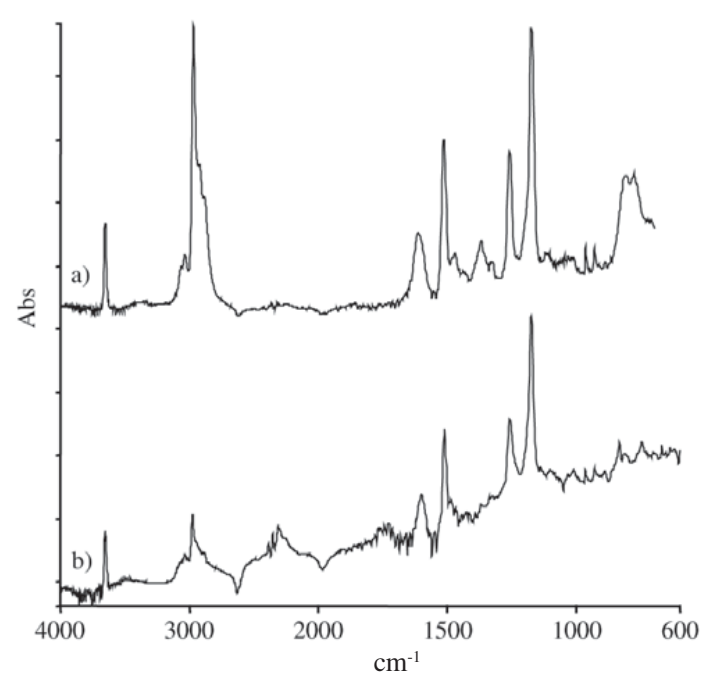

Figura 10. Espectros FT-IR dos produtos de degradação de a) Versamine CEX 13320 e b) SE 3.

quando analisa-se o espectro do produto de degradação de um sistema epoxídico contendo este agente, em comparação ao relativo ao SE 2. As absorções dos dois agentes podem ser observadas.

A Figura 10, de forma similar aos SE anteriores, mostra que é também possível identificar o agente de cura amínico, Versamine CEX 13320 no sistema epoxídico SE3.

O método pode ser avaliado para a investigação de outros tipos de agentes de cura ou endurecedores, segundo adaptações específicas, e é objeto de estudo para publicações posteriores.

\section{Conclusão}

Foi demonstrado que por meio de metodologia CONTROLPIR/FT-IR, desenvolvida em nossos laboratórios, é possível identificar agentes de cura utilizados em resina epoxídica DGEBA, à base de polimercaptana ou aduto de amina em condições adequadas, podendo ser avaliada para a investigação de outros tipos de agentes de cura, segundo adaptações específicas. Por meio do acoplamento TG/FT-IR é possível identificar, além dos agentes de cura à base de polimercaptana e aduto de amina, aqueles que contêm pequeno teor de poliaminoamida em relação ao de polimercaptana.

\section{Referências Bibliográficas}

1. Romão, B.M.V.; Dutra, R.C.L.; Diniz, M.F.; Ribeiro, A.P. Pesquisa Naval, 14, p. 273, 2001.

2. Jiawu, G.; Kui, S.; Mong, G.Z. Termochimica Acta, 352, p. $153,2000$.

3. Sugita, T.; Ito, M. Bull. Chem. Soc. Japan, 38, (10), p. 1620, 1965.

4. Dutra, R.C.L. Modificação de fibras de polipropileno com EVA funcionalizado, Tese de Doutorado, Universidade Federal do Rio de Janeiro, Brasil, 1997.

5. Herrera, M.; Matuschek, G.; Kettrup, A Thermochimica Acta, 361, p. 69, 2000.

6. May, C.A; Hadad D.K.; Browning, C.E. Polym. Eng. Sci., 19, 8, p. 545, 1979.

7. Varley, R.J.; Hawthorne; D.G.; Hodghin, J.H.; Simon, G.P. Polymer, 36 7, p. 1347,1995.

8. Catálogo Cognis‘2001.

9. Urbanski, J.; Czerwinski, W.; Janicka, K.; Majewska, F.; Zowall, H. Handbook of analysis of synthetic polymers and plastics, John Wiley, New York, 1977.

10. Smith, A.L. Applied Infrared Spectroscopy, John Wiley, New York, 1979.

11. Silverstein, R.M.; Bassler, G.C. Spectrometric Identification of Organic Compounds, John Wiley, New York, 1981.

12. Dyakonov, T.; Patrick, J.; Mann, Y.C.; Stevenson, W.T.K. Polymer Degradation and Stability, 54, p. 67, 1996.

13. Reggers, G.; Ruysen, M.; Carleer, R.; Mullens, J. Thermochimica Acta, 295, p. 107, 1997.

14. Hornsby, P.R.; Wang, J.; Rothon, R.; Jackson, G.; Wilkinson, G.; Cossick, K. Polymer Degradation and Stability, 51, p. 235, 1996.

Recebido: 20/12/2002

Aprovado:30/04/2003 